CUBO A Mathematical Journal Vol.13, № 01, (45-60). March 2011

\title{
On the solution of generalized equations and variational inequalities
}

\author{
IOANNIS K. Argyros \\ Cameron University, \\ Department of Mathematics Sciences, \\ Universidad Nacional Autonoma de Mexico, \\ Lawton, OK 73505, USA. \\ email: iargyros@cameron.edu \\ and \\ SAÏD HILOUT \\ Poitiers University, \\ Laboratoire de Mathématiques et Applications, \\ Bd. Pierre et Marie Curie, Téléport 2, B.P. 30179, \\ 86962 Futuroscope Chasseneuil Cedex, France. \\ email: said.hilout@math.univ-poitiers.fr
}

\begin{abstract}
Uko and Argyros provided in [18] a Kantorovich-type theorem on the existence and uniqueness of the solution of a generalized equation of the form $f(u)+g(u) \ni 0$, where $f$ is a Fréchet-differentiable function, and $g$ is a maximal monotone operator defined on a Hilbert space. The sufficient convergence conditions are weaker than the corresponding ones given in the literature for the Kantorovich theorem on a Hilbert space. However, the convergence was shown to be only linear.

In this study, we show under the same conditions, the quadratic instead of the linear convergenve of the generalized Newton iteration involved.
\end{abstract}




\section{RESUMEN}

Uko y Argyros estudian en [18] un teorema tipo-Kantorovich en el existencia y unicidad de la solución de una ecuación generalizada de la forma $f(u)+g(u) \ni 0$, donde $f$ es una función Fréchet-diferenciable, y $g$ es un operador monotono máximo definido en un espacio de Hilbert. Las condiciones de convergencia suficientes son más débiles que los correspondientemente dadas en la literatura para el teorema de Kantorovich en un espacio de Hilbert. Sin embargo, la convergencia ha demostrado ser sólo lineal.

En este estudio, mostramos en las mismas condiciones, la ecuación cuadrática en lugar de la lineal convergente de la iteración generalizada de Newton involucradas.

Keywords: Generalized equation, variational inequality, nonlinear complementarity problem, nonlinear operator equation, Kantorovich theorem, generalized Newton's method, center-Lipschitz condition.

AMS Subject Classification: 65K10, 65J99, 49M15, 49J53, 47J20, 47H04, 90C30, 90C33.

\section{Introduction}

Let $H$ be a Hilbert space, let $C$ be a Let $H$ be a Hilbert space, let $C$ be a closed convex subset of $H$ with non-empty interior $D$, let $f: C \longmapsto H$ be a continuous function that is Fréchet-differentiable on $D$, and let $g$ be a non-empty maximal monotone operator defined on $H \times H$, fixed all through this paper. Then there exists $\alpha \geq 0$ (the monotonicity modulus of $g$ ) such that:

$$
\left[x_{1}, y_{1}\right] \in g \text { and }\left[x_{2}, y_{2}\right] \in g \Longrightarrow\left(y_{2}-y_{1}, x_{2}-x_{1}\right) \geq \alpha\left\|x_{1}-x_{2}\right\|^{2} \text {. }
$$

It is well known (cf. [10]) that $g$ is closed in the sense that

$$
\left[x_{m}, y_{m}\right] \in g, \quad \lim _{m \rightarrow \infty} x_{m}=x \text { and } \quad \lim _{m \rightarrow \infty} y_{m}=y \Longrightarrow[x, y] \in g .
$$

In the sequel, we will regard the statements $[x, y] \in g, g(x) \ni y,-y+g(x) \ni 0$, and $y \in g(x)$ as synonymous. Given any $u_{0} \in D$ and $r>0, \bar{U}\left(u_{0}, r\right)$ will designate the closed ball $\left\{x \in H:\left\|x-u_{0}\right\| \leq r\right\}$, and $U\left(u_{0}, r\right)$ will designate the corresponding open ball.

We are interested in the solvability of the generalized equation:

$$
f(u)+g(u) \ni 0 .
$$

Uko and Argyros provided in [18] a weak Kantorovich theorem that generalizes the Kantorovich theorem for generalized equations, which weakened work by Uko [16] on the Hilbert space 
version of the classical Kantorovich theorem [6, 9] for the solvability of nonlinear operator equations.

The Kantorovich theorem is a fundamental tool in nonlinear analysis for proving the existence and uniqueness of solutions of equations arising in various fields. An important extension of the Kantorovich theorem was obtained recently by Argyros [1], [2], who used a combination of Lipschitz and center-Lipschitz conditions in place of the Lipschitz conditions used by Kantorovich. In the present paper, we will formulate and prove an extension of the Kantorovich theorem for the generalized equation (1.3). The depth and scope of this theorem is such that when we specialize it to nonlinear operator equations we get results that are weaker than the Kantorovich theorem. Our approach will be iterative, and the solution of problem (1.3) will be obtained as the limit of the solutions of the generalized Newton subproblems (GNM):

$$
f^{\prime}\left(u_{m}\right) u_{m+1}+g\left(u_{m+1}\right) \ni f^{\prime}\left(u_{m}\right) u_{m}-f\left(u_{m}\right), \quad m=0,1, \ldots
$$

A well known example (cf. [11]) of a maximal monotone operator is obtained on setting

$$
g(x)=\partial \phi(x) \equiv\{v \in H: \phi(x)-\phi(y) \leq(v, x-y) \quad \forall y \in H\}
$$

where, $\phi: H \longmapsto(-\infty, \infty]$ is a proper lower semicontinuous convex function. In this case problem (1.3) becomes the variational inequality:

$$
f(u)+\partial \phi(u) \ni 0 .
$$

Such problems were introduced in the early sixties by Stampacchia [15] and have found important applications in the physical and engineering sciences and in many other fields [1]-[21].

The generalized Newton iterates (1.4) are obtained as solutions of mildly nonlinear generalized equations of the form:

$$
A z+g(z) \ni b .
$$

In this study, we recover the desired quadratic convergence of the (GNM), not attained in [18] (under the same hypotheses and computational cost).

\section{Semilocal convergence analysis of (GNM)}

Uko and Argyros showed in [18], and with a different approach in [2, Case 3, p. 387] the following result on majorizing sequences for the (GNM):

Lemma 2.1. Let $\eta, \alpha, b, M$ and $M_{0}$ be nonnegative constants. 
Let:

$$
\begin{gathered}
c_{0}>-\alpha, \\
\eta=\frac{b}{c_{0}+\alpha},
\end{gathered}
$$

and

$$
0 \leq M_{0} \leq M
$$

Suppose:

$$
\left(4 M_{0}+M+\sqrt{M^{2}+8 M M_{0}}\right) \leq 4\left(c_{0}+\alpha\right) .
$$

The inequality in (2.1) is strict if $M_{0}=0$.

Then, scalar sequence $\left\{s_{k}\right\}(k \geq 0)$ given by:

$$
s_{0}=0, s_{1}=\eta, s_{k+1}=s_{k}+\frac{M\left(s_{k}-s_{k-1}\right)^{2}}{2\left(1-M_{0} s_{k}\right)}
$$

is well defined, nondecreasing, bounded above by $s^{\star \star}$, and converges to its unique least upper bound $s^{\star} \in\left[0, s^{\star \star}\right]$, where

$$
\begin{gathered}
s^{\star \star}=\frac{2 \eta}{2-\theta}, \\
\theta=\frac{4 M}{M+\sqrt{M^{2}+8 M_{0} M}} \quad\left(M_{0} \neq 0\right) .
\end{gathered}
$$

Moreover, the following estimates hold for all $k \geq 0$ :

$$
\begin{gathered}
M_{0} s^{\star} \leq 1, \\
0 \leq s_{k+1}-s_{k} \leq\left(\frac{\theta}{2}\right)^{k} \eta, \\
0 \leq s^{\star}-s_{k} \leq\left(\frac{\theta}{2}\right)^{k} s^{\star \star} .
\end{gathered}
$$

In the next result, we show that under the same sufficient convergence condition (2.1) for (GNM), we can improve upon the linear error estimates (2.6), and (2.7), and show instead the quadratic convergence of the majorizing sequence $\left\{s_{n}\right\}$.

It is convenient for us to set:

$$
L_{0}=\frac{M_{0}}{c_{0}+\alpha}, \quad \text { and } \quad L=\frac{M}{c_{0}+\alpha} .
$$


Lemma 2.2. Assume there exist constants $L_{0} \geq 0, L \geq 0$, and $\eta \geq 0$, such that:

$$
q_{0}=\bar{L} \eta \leq \frac{1}{2},
$$

where,

$$
\bar{L}=\frac{1}{8}\left(L+4 L_{0}+\sqrt{L^{2}+8 L_{0} L}\right) .
$$

The inequality in (2.8) is strict if $L_{0}=0$.

Then, sequence $\left\{t_{k}\right\}(k \geq 0)$ given by

$$
t_{0}=0, \quad t_{1}=\eta, \quad t_{k+1}=t_{k}+\frac{L_{1}\left(t_{k}-t_{k-1}\right)^{2}}{2\left(1-L_{0} t_{k}\right)} \quad(k \geq 1),
$$

is well defined, nondecreasing, bounded above by $t^{\star \star}$, and converges to its unique least upper bound $t^{\star} \in\left[0, t^{\star \star}\right]$, where

$$
\begin{gathered}
L_{1}=\left\{\begin{array}{lll}
L_{0} & \text { if } & k=1 \\
L & \text { if } & k>1
\end{array},\right. \\
t^{\star \star}=\frac{2 \eta}{2-\delta}, \\
1 \leq \delta=\frac{4 L}{L+\sqrt{L^{2}+8 L_{0} L}}<2 \text { for } L_{0} \neq 0 .
\end{gathered}
$$

Moreover the following estimates hold:

$$
\begin{gathered}
L_{0} t^{\star}<1, \\
0 \leq t_{k+1}-t_{k} \leq \frac{\delta}{2}\left(t_{k}-t_{k-1}\right) \leq \cdots \leq\left(\frac{\delta}{2}\right)^{k} \eta, \quad(k \geq 1), \\
t_{k+1}-t_{k} \leq\left(\frac{\delta}{2}\right)^{k}\left(2 q_{0}\right)^{2^{k}-1} \eta, \quad(k \geq 0), \\
0 \leq t^{\star}-t_{k} \leq\left(\frac{\delta}{2}\right)^{k} \frac{\left(2 q_{0}\right)^{2^{k}-1} \eta}{1-\left(2 q_{0}\right)^{2^{k}}}, \quad\left(2 q_{0}<1\right), \quad(k \geq 0) .
\end{gathered}
$$

Proof. If $L_{0}=0$, then (2.13) holds trivially. In this case, for $L>0$, an induction argument shows that

$$
t_{k+1}-t_{k}=\frac{2}{L}\left(2 q_{0}\right)^{2^{k}} \quad(k \geq 0),
$$

and therefore

$$
t_{k+1}=t_{1}+\left(t_{2}-t_{1}\right)+\cdots+\left(t_{k+1}-t_{k}\right)=\frac{2}{L} \sum_{m=0}^{k}\left(2 q_{0}\right)^{m},
$$


and

$$
t^{\star}=\lim _{k \rightarrow \infty} t_{k}=\frac{2}{L} \sum_{k=0}^{\infty}\left(2 q_{0}\right)^{2^{k}} .
$$

Clearly, this series converges, since $k \leq 2^{k}, 2 q_{0}<1$, and is bounded above by the number

$$
\frac{2}{L} \sum_{k=0}^{\infty}\left(2 q_{0}\right)^{k}=\frac{4}{L(2-L \eta)}
$$

If $L=0$, then in view of $(2.10), 0 \leq L_{0} \leq L$, we deduce: $L_{0}=0$, and $t^{\star}=t_{k}=\eta(k \geq 1)$.

In the rest of the proof, we assume that $L_{0}>0$.

The result until estimate (2.14) follows from Lemma 1 in [2] (see also [1], [3]).

Note that in particular Newton-Kantorovich-type convergence condition (2.8) is given in [2, page 387, Case 3 for $\delta$ given by (2.12). The factor $\eta$ is missing from the left hand side of the inequality three lines before the end of page 387$]$.

In order for us to show (2.15) we need the estimate:

$$
\frac{1-\left(\frac{\delta}{2}\right)^{k+1}}{1-\frac{\delta}{2}} \eta \leq \frac{1}{L_{0}}\left(1-\left(\frac{\delta}{2}\right)^{k-1} \frac{L}{4 \bar{L}}\right) \quad(k \geq 1)
$$

For $k=1,(2.17)$ becomes

$$
\left(1+\frac{\delta}{2}\right) \eta \leq \frac{4 \bar{L}-L}{4 \bar{L} L_{0}}
$$

or

$$
\left(1+\frac{2 L}{L+\sqrt{L^{2}+8 L_{0} L}}\right) \eta \leq \frac{4 L_{0}-L+\sqrt{L^{2}+8 L_{0} L}}{L_{0}\left(4 L_{0}+L+\sqrt{L^{2}+8 L_{0} L}\right)}
$$

In view of (2.8), it suffices to show:

$$
\frac{L_{0}\left(4 L_{0}+L+\sqrt{L^{2}+8 L_{0} L}\right)\left(3 L+\sqrt{L^{2}+8 L_{0} L}\right)}{\left(L+\sqrt{L^{2}+8 L_{0} L}\right)\left(4 L_{0}-L+\sqrt{L^{2}+8 L_{0} L}\right)} \leq 2 \bar{L},
$$

which is true as equality.

Let us now assume estimate (2.17) is true for all integers smaller or equal to $k$. We must show (2.17) holds for $k$ being $k+1$ : 


$$
\frac{1-\left(\frac{\delta}{2}\right)^{k+2}}{1-\frac{\delta}{2}} \eta \leq \frac{1}{L_{0}}\left(1-\left(\frac{\delta}{2}\right)^{k} \frac{L}{4 \bar{L}}\right) \quad(k \geq 1)
$$

or

$$
\left(1+\frac{\delta}{2}+\left(\frac{\delta}{2}\right)^{2}+\cdots+\left(\frac{\delta}{2}\right)^{k+1}\right) \eta \leq \frac{1}{L_{0}}\left(1-\left(\frac{\delta}{2}\right)^{k} \frac{L}{4 \bar{L}}\right) .
$$

By the induction hypothesis to show (2.18), it suffices

$$
\frac{1}{L_{0}}\left(1-\left(\frac{\delta}{2}\right)^{k-1} \frac{L}{4 \bar{L}}\right)+\left(\frac{\delta}{2}\right)^{k+1} \eta \leq \frac{1}{L_{0}}\left(1-\left(\frac{\delta}{2}\right)^{k} \frac{L}{4 \bar{L}}\right)
$$

or

$$
\left(\frac{\delta}{2}\right)^{k+1} \eta \leq \frac{1}{L_{0}}\left(\left(\frac{\delta}{2}\right)^{k-1}-\left(\frac{\delta}{2}\right)^{k}\right) \frac{L}{4 \bar{L}}
$$

or

$$
\delta^{2} \eta \leq \frac{L(2-\delta)}{2 \bar{L} L_{0}}
$$

In view of $(2.8)$ it suffices to show

$$
\frac{2 \bar{L} L_{0} \delta^{2}}{L(2-\delta)} \leq 2 \bar{L}
$$

which holds as equality by the choice of $\delta$ given by (2.12). That completes the induction for estimates (2.17)

We shall show (2.15) using induction on $k \geq 0$ : Estimate (2.15) is true for $k=0$ by (2.8), (2.10), and (2.12). In order for us to show estimate (2.15) for $k=1$, since $t_{2}-t_{1}=\frac{L\left(t_{1}-t_{0}\right)^{2}}{2\left(1-L_{0} t_{1}\right)}$, it suffices:

$$
\frac{L \eta^{2}}{2\left(1-L_{0} \eta\right)} \leq \delta \bar{L} \eta^{2}
$$

or

$$
\frac{L}{1-L_{0} \eta} \leq \frac{16 \bar{L} L}{L+\sqrt{L^{2}+8 L_{0} L}} \quad(\eta \neq 0)
$$

or

$$
\eta \leq \frac{1}{L_{0}}\left(1-\frac{L+\sqrt{L^{2}+8 L_{0} L}}{16 \bar{L}}\right) \quad\left(L_{0} \neq 0, L \neq 0\right) .
$$

But by $(2.8)$

$$
\eta \leq \frac{4}{L+4 L_{0}+\sqrt{L^{2}+8 L_{0} L}}
$$


It then suffices to show

$$
\frac{4}{L+4 L_{0}+\sqrt{L^{2}+8 L_{0} L}} \leq \frac{1}{L_{0}}\left(1-\frac{L+\sqrt{L^{2}+8 L_{0} L}}{16 \bar{L}}\right)
$$

or

$$
\frac{L+\sqrt{L^{2}+8 L_{0} L}}{16 \bar{L}} \leq 1-\frac{4 L_{0}}{L+4 L_{0}+\sqrt{L^{2}+8 L_{0} L}}
$$

or

$$
\frac{L+\sqrt{L^{2}+8 L_{0} L}}{16 \bar{L}} \leq \frac{L+\sqrt{L^{2}+8 L_{0} L}}{L+4 L_{0}+\sqrt{L^{2}+8 L_{0} L}}
$$

or

$$
\bar{L} \geq 0,
$$

which is true by (2.9).

Let us assume (2.18) holds for all integers smaller or equal to $k$. We shall show (2.18) holds for $k$ replaced by $k+1$.

Using (2.10), and the induction hypothesis, we have in turn

$$
\begin{aligned}
t_{k+2}-t_{k+1} & =\frac{L}{2\left(1-L_{0} t_{k+1}\right)}\left(t_{k+1}-t_{k}\right)^{2} \\
& \leq \frac{L}{2\left(1-L_{0} t_{k+1}\right)}\left(\left(\frac{\delta}{2}\right)^{k}\left(2 q_{0}\right)^{2^{k}-1} \eta\right)^{2} \\
& \leq \frac{L}{2\left(1-L_{0} t_{k+1}\right)}\left(\left(\frac{\delta}{2}\right)^{k-1}\left(2 q_{0}\right)^{-1} \eta\right)\left(\left(\frac{\delta}{2}\right)^{k+1}\left(2 q_{0}\right)^{2^{k+1}-1} \eta\right) \\
& \leq\left(\frac{\delta}{2}\right)^{k+1}\left(2 q_{0}\right)^{2^{k+1}-1} \eta,
\end{aligned}
$$

since,

$$
\frac{L}{2\left(1-L_{0} t_{k+1}\right)}\left(\left(\frac{\delta}{2}\right)^{k-1}\left(2 q_{0}\right)^{-1} \eta\right) \leq 1, \quad(k \geq 1) .
$$

Indeed, we can show instead of (2.19):

$$
t_{k+1} \leq \frac{1}{L_{0}}\left(1-\left(\frac{\delta}{2}\right)^{k-1} \frac{L}{4 \bar{L}}\right),
$$

which is true, since by (2.14), and the induction hypothesis: 


$$
\begin{aligned}
t_{k+1} & \leq t_{k}+\frac{\delta}{2}\left(t_{k}-t_{k-1}\right) \\
& \leq t_{1}+\frac{\delta}{2}\left(t_{1}-t_{0}\right)+\cdots+\frac{\delta}{2}\left(t_{k}-t_{k-1}\right) \\
& \leq \eta+\left(\frac{\delta}{2}\right) \eta+\cdots+\left(\frac{\delta}{2}\right)^{k} \eta \\
& =\frac{1-\left(\frac{\delta}{2}\right)^{k+1}}{1-\frac{\delta}{2}} \eta \\
& \leq \frac{1}{L_{0}}\left(1-\left(\frac{\delta}{2}\right)^{k-1} \frac{L}{4 \bar{L}}\right)
\end{aligned}
$$

That completes the induction for estimate (2.15).

Using estimate (2.18) for $j \geq k$, we obtain in turn for $2 q_{0}<1$ :

$$
\begin{aligned}
t_{j+1}-t_{k} & =\left(t_{j+1}-t_{j}\right)+\left(t_{j}-t_{j-1}\right)+\cdots+\left(t_{k+1}-t_{k}\right) \\
& \leq\left(\left(\frac{\delta}{2}\right)^{j}\left(2 q_{0}\right)^{2^{j}-1}+\left(\frac{\delta}{2}\right)^{j-1}\left(2 q_{0}\right)^{2^{j-1}-1}+\cdots+\left(\frac{\delta}{2}\right)^{k}\left(2 q_{0}\right)^{2^{k}-1}\right) \eta \\
& \leq\left(1+\left(2 q_{0}\right)^{2^{k}}+\left(\left(2 q_{0}\right)^{2^{k}}\right)^{2}+\cdots\right)\left(\frac{\delta}{2}\right)^{k}\left(2 q_{0}\right)^{2^{k}-1} \eta \\
& =\left(\frac{\delta}{2}\right)^{k} \frac{\left(2 q_{0}\right)^{2^{k}-1} \eta}{1-\left(2 q_{0}\right)^{2^{k}}} .
\end{aligned}
$$

Estimate (2.16) follows from (2.20) by letting $j \longrightarrow \infty$.

That completes the proof of Lemma 2.2 .

We need the result [18], [3],[10], [11].

Lemma 2.3. Let $g$ be a maximal monotone operator satisfying condition (1.1), and let $A$ be a bounded linear operator mapping $H$ into $H$. If there exists $c \in \mathbb{R}$, such that $c>-\alpha$, and

$$
(A x, x) \geq c\|x\|^{2}, \quad \forall x \in H .
$$

Then, for any $b \in H$, the problem

$$
A z+g(z) \ni b
$$

has a unique solution $z \in H$.

We now use Lemma 2.2 instead of Lemma 2.1 to improve, in particular the error estimates, in the weak Kantorovich-type existence theorem for problem (1.3) given in [18]. 
Theorem 2.1. Let $g$ be a maximal monotone operator satisfying condition (1.1), and suppose that there exist $u_{0} \in D$, and $v_{0} \in H$, such that

$$
\begin{aligned}
v_{0} \in g\left(u_{0}\right) & \\
\left(f^{\prime}\left(u_{0}\right) x, x\right) & \geq c_{0}\|x\|^{2}, \quad \forall x \in H, \\
\left\|f^{\prime}(x)-f^{\prime}(y)\right\| & \leq M\|x-y\| \quad \forall x, y \in D, \\
\left\|f^{\prime}(x)-f^{\prime}\left(u_{0}\right)\right\| & \leq M_{0}\left\|x-u_{0}\right\| \quad \forall x \in D, \\
\left\|f\left(u_{0}\right)+v_{0}\right\| & \leq b,
\end{aligned}
$$

where $b \geq 0, M \geq M_{0} \geq 0$ and $c_{0}>-\alpha$.

Let

$$
\eta=\frac{b}{c_{0}+\alpha},
$$

and suppose condition (2.8), and $\bar{U}\left(u_{0}, t^{\star}\right) \subseteq D$ hold, where the $t^{\star}$ is the limit of the sequence $\left\{t_{m}\right\}$ defined in Lemma 2.2.

Then equation (1.3) has a unique solution $u$ in $\bar{U}\left(u_{0}, r\right)$, where

$$
r=\frac{2 \eta}{1+\sqrt{c_{0}+\alpha-2 M_{0} \eta}},
$$

and the Newton iterations generated from (GNM) converge to u, and satisfy the estimates:

$$
\begin{aligned}
\left\|u_{m}-u_{m-1}\right\| & \leq t_{m}-t_{m-1}, \\
\left\|u_{m}-u_{0}\right\| & \leq t_{m}, \\
\left\|u-u_{m}\right\| & \leq t^{\star}-t_{m} .
\end{aligned}
$$

Moreover, if there exists $T \geq 0$, such that:

$$
M_{0}(r+T)<2\left(c_{0}+\alpha\right),
$$

then, the solution is unique in $\bar{U}\left(u_{0}, T\right) \cap D$. This solution is also unique in the sets $\bar{U}\left(u_{0}, t^{\star}\right)$, and $\bar{U}\left(u_{0}, r\right) \cup\left(D \cap U\left(u_{0}, R\right)\right)$, where

$$
R=\frac{2\left(c_{0}+\alpha\right)}{M_{0}-r}
$$

Furthemore, estimates (2.14)-(2.16) hold.

\section{Proof.}

It follows from condition (2.23), and Lemma 2.2, that the first iterate $u_{1}$ is defined uniquely in (1.4). Using (1.4), (2.22), and the monotonicity condition (1.1), we obtain

$$
\alpha\left\|u_{1}-u_{0}\right\|^{2}+\left(v_{0}+f\left(u_{0}\right)+f^{\prime}\left(u_{0}\right)\left(u_{1}-u_{0}\right), u_{1}-u_{0}\right) \leq 0 .
$$


Rewriting this in the form

$$
\alpha\left\|u_{1}-u_{0}\right\|^{2}+\left(f^{\prime}\left(u_{0}\right)\left(u_{1}-u_{0}\right), u_{1}-u_{0}\right) \leq\left(-f\left(u_{0}\right)-v_{0}, u_{1}-u_{0}\right)
$$

and making use of (2.23) and (2.26), we see that:

$$
\left\|u_{1}-u_{0}\right\| \leq \frac{b}{c_{0}+\alpha}=\eta=t_{1}-t_{0} .
$$

If $M_{0}=0$, then $r=\eta \leq t^{\star}$, and $R=\infty$. In this case $f^{\prime}(x)=f^{\prime}\left(u_{0}\right)$, for all $x \in D$, and

$$
\begin{aligned}
f(x) & =f\left(u_{0}\right)+\int_{0}^{1} f^{\prime}\left(s x+(1-s) u_{0}\right)\left(x-u_{0}\right) d s \\
& =f\left(u_{0}\right)+f^{\prime}\left(u_{0}\right)\left(x-u_{0}\right), \quad \forall x \in D .
\end{aligned}
$$

Therefore, the unique solution of equation (1.3) in $D$ is $u=u_{1}$. Since $\left\|u_{1}-u_{0}\right\| \leq \eta \leq t^{\star}$, the conclusion of the theorem holds in this case. If $\eta=0$, then $r=t^{\star}=0, R=\frac{2\left(c_{0}+\alpha\right)}{M_{0}}$, and the unique solution of equation (1.3) in $\bar{U}\left(u_{0}, r\right) \cup\left(D \cap U\left(u_{0}, R\right)\right)$ is $u=u_{0}$.

In the rest of the proof, we assume that $M_{0}>0$, and $\eta>0$. In this case, it follows from (2.8) that:

$$
2 M_{0} \leq \frac{4 M_{0}+M+\sqrt{M^{2}+8 M M_{0}}}{4} \leq \frac{c_{0}+\alpha}{\eta} .
$$

Therefore, $r$ and $R$ are well defined.

We prove by induction that the $u_{m}$ are well defined and conditions (2.28) and (2.29) hold for $m=0,1, \ldots$ It follows from $(2.32)$, that the induction hypothesis is true if $m=1$.

We assume that $m \geq 1$, and that the induction hypothesis holds for $m$. Then it follows from (2.25) and (2.29), that

$$
\left\|f^{\prime}\left(u_{m}\right)-f^{\prime}\left(u_{0}\right)\right\| \leq M_{0}\left\|u_{m}-u_{0}\right\| \leq M_{0} t_{m} .
$$

Therefore

$$
\left(f^{\prime}\left(u_{0}\right) x-f^{\prime}\left(u_{m}\right) x, x\right) \leq\left\|f^{\prime}\left(u_{0}\right)-f^{\prime}\left(u_{m}\right)\right\|\|x\|^{2} \leq M_{0} t_{m}\|x\|^{2}
$$

for all $x \in H$, which implies, because of (2.23), that:

$$
\left(f^{\prime}\left(u_{m}\right) x, x\right) \geq\left(c_{0}-M_{0} t_{m}\right)\|x\|^{2}, \quad \forall x \in H .
$$


On the other hand, it follows from (1.4), that $c_{0}-M_{0} t_{m}>-\alpha$. Therefore we conclude from Lemma 2.2 , that the iterate $u_{m+1}$ is defined uniquely in (1.4).

It follows from (1.4) that

$$
g\left(u_{m}\right) \ni v_{m} \equiv-f\left(u_{m-1}\right)-f^{\prime}\left(u_{m-1}\right)\left(u_{m}-u_{m-1}\right)
$$

and by using conditions (2.24) and (2.28), we see that

$$
\begin{aligned}
\left\|f\left(u_{m}\right)+v_{m}\right\| & =\left\|f\left(u_{m}\right)-f\left(u_{m-1}\right)-f^{\prime}\left(u_{m-1}\right)\left(u_{m}-u_{m-1}\right)\right\| \\
& =\left\|\int_{0}^{1}\left[f^{\prime}\left((1-s) u_{m-1}+s u_{m}\right)-f^{\prime}\left(u_{m-1}\right)\right]\left(u_{m}-u_{m-1}\right) d s\right\| \\
& \leq \frac{M}{2}\left\|u_{m}-u_{m-1}\right\|^{2} \leq \frac{M}{2}\left(t_{m}-t_{m-1}\right)^{2} .
\end{aligned}
$$

On the other hand, it follows from (1.1), (2.34), and (1.4) that:

$$
\alpha\left\|u_{m+1}-u_{m}\right\|^{2}+\left(v_{m}+f\left(u_{m}\right)+f^{\prime}\left(u_{m}\right)\left(u_{m+1}-u_{m}\right), u_{m+1}-u_{m}\right) \leq 0 .
$$

Rewriting this in the form

$$
\alpha\left\|u_{m+1}-u_{m}\right\|^{2}+\left(f^{\prime}\left(u_{m}\right)\left(u_{m+1}-u_{m}\right), u_{m+1}-u_{m}\right) \leq\left(f\left(u_{m}\right)+v_{m}, u_{m}-u_{m+1}\right)
$$

and making use of (2.33) and (2.35), we see that

$$
\left\|u_{m+1}-u_{m}\right\| \leq \frac{M\left(t_{m}-t_{m-1}\right)^{2}}{2\left(c_{0}+\alpha-M_{0} t_{m}\right)}=t_{m+1}-t_{m}
$$

Hence $\left\|u_{m+1}-u_{0}\right\| \leq\left\|u_{m+1}-u_{m}\right\|+\left\|u_{m}-u_{0}\right\| \leq t_{m+1}-t_{m}+t_{m}=t_{m+1}$. It follows that (2.28) and (2.29) also hold when $m$ is replaced with $m+1$ and hence, by induction, that they hold for all positive integral values of $m$.

This implies that

$$
\left\|u_{m+q}-u_{m}\right\| \leq \sum_{k=m+1}^{m+q}\left\|u_{k}-u_{k-1}\right\| \leq \sum_{k=m+1}^{m+q}\left(t_{k}-t_{k-1}\right)=t_{m+q}-t_{m} .
$$

Since $\left\{t_{m}\right\}$ is a Cauchy sequence, it follows that $\left\{u_{m}\right\}$ is also a Cauchy sequence converging to some $u \in \bar{U}\left(u_{0}, t^{\star}\right)$. On letting $q$ tend to infinity we see that $(2.30)$ holds. Since $\lim _{m \rightarrow \infty} v_{m}=-f(u)$ and $\lim _{m \rightarrow \infty} u_{m}=u$, it follows from (2.34), and property (1.2) that $u$ solves problem (1.3). 
To prove the uniqueness assertions, let $v$ be any solution of problem (1.3). Then since $-f(v) \in$ $g(v)$, it follows from (1.2), (2.34), and (2.22) that:

$$
\begin{aligned}
\alpha\left\|v-u_{0}\right\|^{2} & \leq-\left(v_{0}+f(v), v-u_{0}\right) \\
& =-\left(f(v)-f\left(u_{0}\right)-f^{\prime}\left(u_{0}\right)\left(v-u_{0}\right), v-u_{0}\right) \\
& -\left(f^{\prime}\left(u_{0}\right)\left(v-u_{0}\right), v-u_{0}\right)-\left(f\left(u_{0}\right)+v_{0}, v-u_{0}\right) .
\end{aligned}
$$

Therefore, by (2.23), and the center-Lipschitz condition (2.25), we get:

$$
\begin{aligned}
\left(c_{0}+\alpha\right)\left\|v-u_{0}\right\| & \leq\left\|f(v)-f\left(u_{0}\right)-f^{\prime}\left(u_{0}\right)\left(v-u_{0}\right)\right\|+\left\|f\left(u_{0}\right)+v_{0}\right\| \\
& \leq\left\|\int_{0}^{1}\left[f^{\prime}\left(s v+(1-s) u_{0}\right)-f^{\prime}\left(u_{0}\right)\right]\left(v-u_{0}\right) d s\right\|+b \\
& \leq M_{0}\left\|v-u_{0}\right\| \int_{0}^{1}\left(s\left\|v-u_{0}\right\|+(1-s)\left\|v-u_{0}\right\|\right) d s+b \\
& =M_{0} \frac{\left\|v-u_{0}\right\|^{2}}{2}+b .
\end{aligned}
$$

By solving this quadratic inequality, we see that either

$$
\left\|v-u_{0}\right\| \leq r \quad \text { or } \quad\left\|v-u_{0}\right\| \geq R .
$$

In particular case $v=u$, condition (2.13) implies that the condition $\left\|u-u_{0}\right\| \geq R$ cannot hold since $\left\|u-u_{0}\right\| \leq t^{\star} \leq \frac{c_{0}+\alpha}{M_{0}}<R$. It follows thet $\left\|u-u_{0}\right\| \geq r$, and hence $u \in \bar{U}\left(u_{0}, r\right)$.

If condition (2.31) holds, and $v$ is a solution of problem (1.3) in $D \cap \bar{U}\left(u_{0}, r\right)$. Then, since $-f(u) \in g(u)$, and $-f(v) \in g(v)$, it follows from (1.2) that:

$$
\begin{aligned}
\alpha\|u-v\|^{2} & \leq(f(v)-f(u), u-v) \\
& =-\left(f(u)-f(v)-f^{\prime}\left(u_{0}\right)(u-v), u-v\right)-\left(f^{\prime}\left(u_{0}\right)(u-v), u-v\right) .
\end{aligned}
$$

If we now apply (2.23), and the center-Lipschitz condition (2.25), we see that

$$
\begin{aligned}
\left(c_{0}+\alpha\right)\|u-v\| & \leq\left\|f(u)-f(v)-f^{\prime}\left(u_{0}\right)(u-v)\right\| \\
& \leq\left\|\int_{0}^{1}\left[f^{\prime}(s u+(1-s) v)-f^{\prime}\left(u_{0}\right)\right](u-v) d s\right\| \\
& \leq M_{0}\|u-v\| \int_{0}^{1}\left(s\left\|u-u_{0}\right\|+(1-s)\left\|v-u_{0}\right\|\right) d s \\
& =M_{0}\|u-v\| \frac{\left\|u-u_{0}\right\|+\left\|v-u_{0}\right\|}{2} \\
& \leq M_{0}\|u-v\| \frac{r+T}{2} .
\end{aligned}
$$


Therefore, it follows from (2.31) that $u=v$. This proves the uniqueness of the solution in $\bar{U}\left(u_{0}, T\right) \cap D$. If we set $T=t^{\star}$, then condition (2.31) reduces to the condition $M_{0} t^{\star}<$ $c_{0}+\alpha+\sqrt{c_{0}+\alpha-2 M_{0} \eta}$, which is true by (2.8). This shows that the solution of problem (1.3) is unique in $\bar{U}\left(u_{0}, t^{\star}\right) \cap D$.

Let

$$
r_{0}=0, \quad r_{1}=\eta, \quad r_{m+1}=r_{m}+\frac{M_{0}\left(r_{m}-r_{m-1}\right)^{2}}{2\left(c_{0}+\alpha-M_{0} r_{m}\right)},
$$

then, the argument employed in the proof of Lemma 2.2 shows that $r_{m}$ converges monotonically to a non-negative number $r$, such that $M_{0} r \leq c_{0}+\alpha$. Since

$$
\begin{aligned}
2\left(c_{0}+\alpha\right) r_{m+1}-2 M_{0} r_{m+1} r_{m} & =2\left(c_{0}+\alpha\right) r_{m}-2 M_{0} r_{m} r_{m-1} \\
& =\cdots \\
& =2\left(c_{0}+\alpha\right) r_{1}-2 M_{0} r_{1} r_{0}=2 \eta\left(c_{0}+\alpha\right) .
\end{aligned}
$$

By letting $m \longrightarrow \infty$, we obtain that $r$ satisfies the equation

$$
-2\left(c_{0}+\alpha\right) r+M_{0} r^{2}+2 \eta\left(c_{0}+\alpha\right)=0 .
$$

Therefore, $r$ is given by the expression (2.27). On the other hand, it follows from (2.10), and an easy induction argument that the inequality $r_{m} \leq t_{m}$ holds for all $m$. This shows that $r \leq t^{\star}$, and we conclude that the solution of problem (1.3) is unique in $\bar{U}\left(u_{0}, r\right) \cup\left(D \cap U\left(u_{0}, R\right)\right)$.

That completes the proof of Theorem 2.1.

We complete this study with a numerical example.

Example 1 . Let $\mathcal{X}=\mathcal{Y}=\mathbb{R}, D=\left(\frac{8}{9}, \frac{10}{9}\right), u_{0}=1$, and define function $F$ on $D$ by

$$
F(x)=6 x^{3}-1
$$

Set $g=0, f(x)=F^{\prime}\left(u_{0}\right)^{-1} F(x)$, then we have

$$
f^{\prime}\left(u_{0}\right)=I, \quad \alpha=0, \quad c_{0}=1, \quad M_{0}=1.9, \quad M=2, \quad \eta=.258646 .
$$

The classical Newton-Kantorovich condition [1]-[3], [5], [6], [9]:

$$
M \eta \leq \frac{1}{2}
$$

is violated, since

$$
M \eta=.517292>.5 \text {. }
$$


However, condition (2.1) becomes

$$
3.999999528<4
$$

Condition (2.36) implies (2.1), but not necessarily vice verca unless if $M=M_{0}$.

Note also that:

$$
M_{0} \leq M
$$

holds in general, and $\frac{M}{M_{0}}$ can be arbitrarily large [2], [3].

We also have:

$$
\begin{gathered}
\theta=\delta=1.017145084, \quad \frac{\theta}{2}=.508572542, \quad s^{\star \star}=.526315727 \\
q_{0}=.499999941, \quad 2 q_{0}=.999999882 .
\end{gathered}
$$

By comparing (2.6) with (2.15), we see that is an improvment by $\left(2 q_{0}\right)^{2^{k}-1}$ at each $k$-th-step.

Several special cases, and other applications can also be found in [18] (see also [1]-[17], [19], $[20])$.

Received: October 2009. Revised: November 2009.

\section{References}

[1] Argyros, I.K.,On the Newton-Kantorovich hypothesis for solving equations, J. Comput. Appl. Math., 11 (2004), 103-110.

[2] Argyros, I.K.,A unifying local-semilocal convergence analysis and applications for twopoint Newton-like methods in Banach space, J. Math. Anal. Appl., 298 (2004), 374-397.

[3] Argyros, I.K.,Computational Theory for iterative methods, Studies in Computational Mathematics, Editors: C.K. Chui and L. Wyutack, Volume 15, Elsevier, New York, USA, 2007.

[4] Bonnans, J.F.,Local analysis of Newton-type methods for variational inequalities and nonlinear programming, Appl. Math. Optimiz., 29 (1994), 161-186.

[5] Dennis, J.E., On the Kantorovich hypotheses for Newton's method, SIAM J. Numer. Anal., 6 (1969), 493-507. 
[6] Gragg, W.B., Tapia, R.A.,Optimal error bounds for the Newton-Kantorovich theorem, SIAM J. Numer. Anal., 11 (1974), 10-13.

[7] Harker, P.T., PAng, J.S.,Finite dimensional variational inequality and nonlinear complementarity problems: a survey of theory, algorithms and applications, Math. Programming 48 (1990), 161-220.

[8] Josephy, N.H.,Newton's method for generalized equations, Technical Report No. 1965, Mathematics Research Center, University of Wisconsin (Madision, WI, 1979).

[9] Kantorovich, L.V.,On Newton's method for functional equations (Russian), Doklady Akademii Nauk SSSR, 59 (1948), 1237-1240.

[10] Minty, G.J.,Monotone (nonlinear) operators in Hilbert space, Duke Math. J., 29 (1973), $341-346$.

[11] Minty, G.J.,On the monotonicity of the gradient of a convex function, Pacific J. Math., 14 (1964), 243-247.

[12] Robinson, S.M.,Strongly regular generalized equations, Math. Oper. Research, 5 (1980), $43-62$.

[13] Robinson, S.M.,Generalized equations, in: A. Bachem, M. Grŏtschel and B. Korte, eds., Math. programming: the state of the art (Springer, Berlin, 1982), 346-367.

[14] Solodov, M.V., Svaiter, B.F.,A truly globally convergent Newton-type method for the monotone nonlinear complementarity problem, SIAM J. Optim., 10 (2000), 605-625.

[15] Stampacchia, G.,Formes bilinéaires coercitives sur les ensembles convexes, C.R.A.S. de Paris, 258 (1964), 4413-4416.

[16] Uko, L.U.,Generalized equations and the generalized Newton method, Math. Programming, 73 (1996), 251-268.

[17] Uko, L.U.,On a class of general strongly nonlinear quasivariational inequalities, Revista di Matematica Pura ed Applicata, 11 (1992), 47-55.

[18] Uko, L.U., Argyros, I.K.,Generalized equations, variational inequalities, and a weak Kantorovich theorem, Numerical Algorithms, doi: 10.1007/s11075-009-9275-2.

[19] Uko, L.U., Orozco, J.C.,Some $p$-norm convergence results for Jacobi and Gauss-Seidel iterations, Revista Colombiana de Matemáticas, 38 (2004), 65-71.

[20] Verma, R.U.,A class of projection-contraction methods applied to monotone variational inequalities, Appl. Math. Lett., 13 (2000), 55-62.

[21] Wang, Z., Shen, Z.,Kantorovich theorem for variational inequalities, Applied Mathematics and Mechanics (English Edition), 25 (2004), 1291-1297. 